\title{
Two loop lattice expansion of the Schrödinger functional coupling in improved QCD
}

\author{
A. Bode ${ }^{a}$, P. Weisz ${ }^{b}$ and U. Wolff ${ }^{c}$ \\ ${ }^{a}$ SCRI, Florida State University, \\ Tallahassee, Fl, 32306-4130, USA \\ ${ }^{b}$ Max-Planck-Institut für Physik, Föhringerring 6, \\ D-80805 München, Germany \\ ${ }^{c}$ Institut für Physik, Humboldt Universität, Invalidenstr. 110, \\ D-10099 Berlin, Germany
}

November 13, 2018

\begin{abstract}
The contributions of the improved fermion action of Sheikholeslami and Wohlert to the two loop coefficient of the expansion of the Schrödinger functional coupling in terms of the lattice coupling are calculated for the gauge group SU(3). These coefficients are required for the second order relation of lattice data to the $\overline{\mathrm{MS}}$-coupling. By taking into account all improvement coefficients we are able to improve the Schrödinger functional to two loop order. This article is based on the revised version of [9].
\end{abstract}

\section{Introduction}

In the framework of the Schrödinger functional the renormalized coupling $\alpha_{\mathrm{SF}}$ can be traced from low to high energy numerically on the lattice with finite size techniques. At high energy a conversion to $\alpha \overline{\mathrm{MS}}$ with perturbation theory is possible. The relation can be calculated by expanding both $\alpha_{\overline{\mathrm{MS}}}$ and $\alpha_{\mathrm{SF}}$ in $g_{0}$, the lattice bare coupling.

This program has been completed for the pure gauge sector $\left(N_{\mathrm{f}}=0\right)$ of QCD [1] where the two loop relation between $\alpha_{\overline{\mathrm{MS}}}$ and $\alpha_{\mathrm{SF}}$ was required in order to avoid a significant source of error in the conversion to $\alpha_{\overline{\mathrm{MS}}}$. Here we present the perturbative two loop relation for arbitrary $N_{\mathrm{f}}$ for the mass-independent scheme described in [2]. 
The outline of the calculation is analogous to the pure gauge case [3]. We have to calculate the perturbative coefficients depending on the box size $I=$ $L / a$, where $\alpha_{\mathrm{SF}}$ is defined. From this we are able to extract the continuum results and the $\mathrm{O}(a)$ improvement terms.

\section{Definition of $\alpha_{\mathrm{SF}}$}

The coupling is defined via the effective action $\Gamma$ in a finite box with extension L

$$
\exp (-\Gamma)=\int D[U] D[\bar{\psi}] D[\psi] \exp (-S[U, \bar{\psi}, \psi])
$$

where $S$ consists of the Wilson gauge action and Sheikholeslami-Wohlert fermion action. In the spacelike directions periodic boundary conditions with an additional phase for the fermions are used. The timelike boundaries are given by a constant and diagonal colour field depending on the parameter $\eta$. Certain $1 \pm \gamma_{0}$ projected fermion field components are set to zero at $t=0, L$. To achieve $\mathrm{O}(a)$ improvement we have to add coupling dependent weights to some links

and plaquettes on the boundaries. Details of the definition of the boundaries can be found in 4 .

The coupling $\alpha_{\mathrm{SF}}(q)=g_{\mathrm{SF}}^{2}(L) /(4 \pi)$, at $q=L^{-1}$,

$$
g_{\mathrm{SF}}^{2}(L)=\left.\frac{\Gamma_{0}^{\prime}(L, \eta)}{\Gamma^{\prime}(L, \eta)}\right|_{\eta=0}
$$

is normalised via the classical action minimal $\Gamma_{0}$. The structure of the expansion is given by

$$
\begin{aligned}
g_{\mathrm{SF}}^{2}(L)= & g_{0}^{2}+\left[p_{10}(I)+p_{11}(I) N_{\mathrm{f}}\right] g_{0}^{4} \\
& +\left[p_{20}(I)+p_{21}(I) N_{\mathrm{f}}+p_{22}(I) N_{\mathrm{f}}^{2}\right] g_{0}^{6}+\mathrm{O}\left(g_{0}^{8}\right)
\end{aligned}
$$

where the dependence on the one and two loop improvement coefficients is hidden in $p_{i j}$. The $p_{i j}$ are sums of Feynman diagrams. The coefficients presented here are $p_{21}(I)$ and $p_{22}(I)$. The one loop coefficient $p_{11}(I)$ was computed in [5].

\section{Perturbative expansion}

Since the classical action minimum is not affected by the presence of the fermions we can use the gluon and ghost propagators of the pure gauge case. The fermion propagators must be calculated numerically. A recurrence scheme similar to the other propagators is developed and will be described elsewhere [6]. The 9 diagrams involving fermions are summed in position space.

Several discrete symmetries can be used to reduce the number of terms. The computational complexity and resulting memory requirements increase by 
including the fermions due to the Dirac trace. Hence we coded two versions, one where we store all components of the Dirac propagator and traces are easy to evaluate and one with a reduced set of spinor components (due to the cubic symmetry in space) but slightly more complicated Dirac traces.

Careful tests were applied: the symmetries of propagators, diagrams and results, gauge parameter independence of $p_{2 j}(I)$, comparison of results for small $L$ with an independently written program using momentum space sums, verification of the universal $\beta$-function coefficients, consistency relations of Symanzik improvement.

In order to get the continuum relation we have to extract various (sub)leading coefficients of the asymptotic series in $a / L$ describing $p_{2 i}$. Apart from the well known blocking procedure an alternative method has been developed and will be described in [6]. Since further development is in progress and further data are being generated, all results are preliminary.

This gives up to $\mathrm{O}\left(\ln ^{2}(I) / I^{2}\right)$ :

$$
\begin{aligned}
& p_{10}(I)=2 b_{00} \ln (I)+0.36828215(13), \\
& p_{11}(I)=2 b_{01} \ln (I)-0.034664940(4), \\
& p_{20}(I)=2 b_{10} \ln (I)+0.048085(63)+p_{10}(I)^{2}, \\
& p_{21}(I)=2 b_{11} \ln (I)-0.004738(56)+2 p_{10}(I) p_{11}(I), \\
& p_{22}(I)=0.000211(29)+p_{11}(I)^{2} .
\end{aligned}
$$

All terms $\propto \ln ^{i}(I) / I$ are zero due to improvement. The fermionic contributions to the boundary improvement coefficients $c_{\mathrm{t}}^{(2)}$ are also determined. In addition the expansion coefficients for the standard Wilson action $c_{\mathrm{sw}}=0$, are calculated. This is numerically cheap because the clover vertices dominate the calculation.

\section{Evolution of $\alpha_{\mathrm{SF}}$}

The non-perturbative evolution of the coupling $\alpha_{\mathrm{SF}}$ for $N_{\mathrm{f}}=0$ [7] and the perturbative three loop evolution for $N_{\mathrm{f}}=0,2$ is shown in figure 1 . These curves are integrated with the smallest $\alpha_{\mathrm{SF}}$ value as initial value (the absolute scale applies only for $N_{\mathrm{f}}=0$ ). From perturbation theory we can expect a visible shift in the evolution of the coupling going from $N_{\mathrm{f}}=0$ to $N_{\mathrm{f}}=2$. 


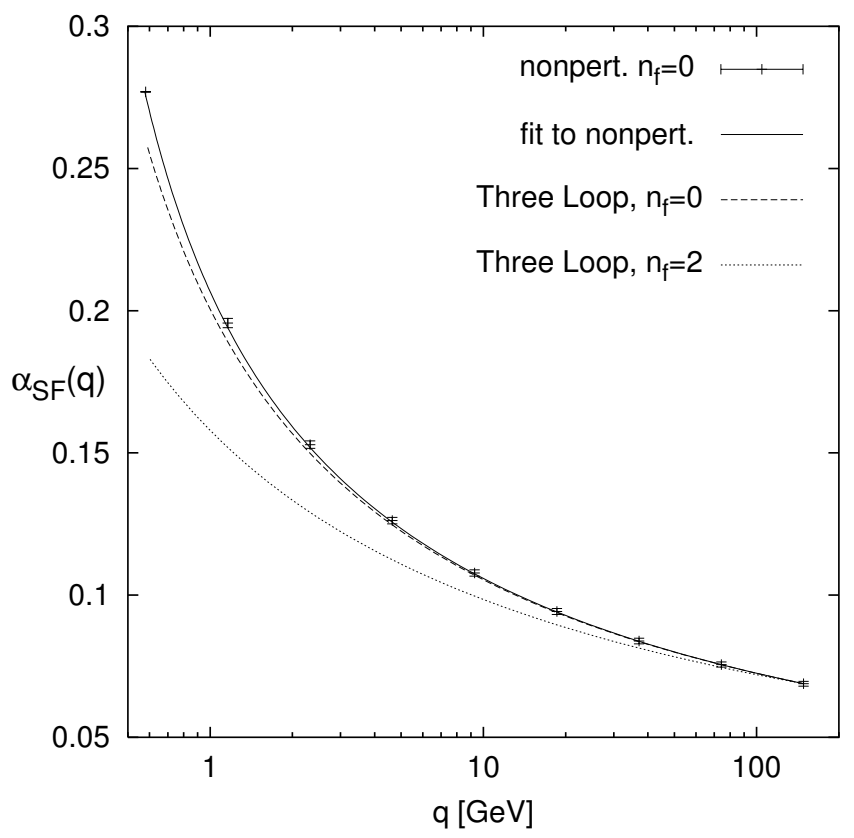

Figure 1: Evolution of the coupling $\alpha_{\mathrm{SF}}$.

\section{Connections to other couplings}

Up to now $\alpha \overline{\mathrm{MS}}$ in $\alpha_{0}$ is known to two loop order only for the unimproved action, $c_{\mathrm{sw}}=0$ [8, 9]. Connecting the series for $N_{\mathrm{f}}=2$ gives

$$
\begin{aligned}
& \alpha \overline{\mathrm{MS}}(s q)=\alpha_{\mathrm{SF}}+c_{1}(s) \alpha_{\mathrm{SF}}(q)^{2}+c_{2}(s) \alpha_{\mathrm{SF}}(q)^{3}, \\
& c_{1}(s)=-8 \pi b_{0} \ln (s)+1.3353 \\
& c_{2}(s)=c_{1}(s)^{2}-32 \pi^{2} b_{1} \ln (s)+3.13(3) .
\end{aligned}
$$

With $b_{2}^{\overline{\mathrm{MS}}}$ known we are able to quote

$$
b_{2}^{\mathrm{SF}}(4 \pi)^{3}=0.482(7)-0.275(8) N_{\mathrm{f}}+0.036(2) N_{\mathrm{f}}^{2}-0.00175(8) N_{\mathrm{f}}^{3}
$$

which results in $b_{2}^{\mathrm{SF}}=0.063(24) /(4 \pi)^{3}$ for $N_{\mathrm{f}}=2$. The perturbative relation (9) between $\alpha_{\mathrm{SF}}$ and $\alpha_{\overline{\mathrm{MS}}}$ involves the scale factor $s$. Fixing it by demanding either $c_{1}(s)=0$ or $c_{2}(s)$ to be minimum gives comparable results:

$$
\begin{aligned}
& \alpha_{\overline{\mathrm{MS}}}(2.3820 q)=\alpha_{\mathrm{SF}}(q)+0.50(3) \alpha_{\mathrm{SF}}(q)^{3}, \\
& \alpha_{\overline{\mathrm{MS}}}(2.9243 q)=\alpha_{\mathrm{SF}}(q)-0.3156 \alpha_{\mathrm{SF}}(q)^{2}+0.40(3) \alpha_{\mathrm{SF}}(q)^{3},
\end{aligned}
$$

where the quantities quoted without error are in all digits significant. The large two loop coefficients might require smaller couplings for a conversion to obtain the same error as for the $N_{\mathrm{f}}=0$ case. 
The two loop relation between the lattice couplings for the improved theory and the Wilson fermion action and the corresponding three loop $\beta$-function coefficients are (for $N=3$ ) also available.

\section{$6 \quad$ Finite $a$ effects}

In numerical simulation the finite $a$ step scaling function

$$
g_{\mathrm{SF}}^{2}(s L)=\Sigma\left(s, g_{\mathrm{SF}}^{2}(L), a / L\right)
$$

is accessible, and the continuum limit

$$
\sigma\left(s, g_{\mathrm{SF}}^{2}(L)\right)=\lim _{a \rightarrow 0} \Sigma\left(s, g_{\mathrm{SF}}^{2}(L), a / L\right)
$$

has to be taken. The perturbative finite $a$ effects are defined from the series $p_{i j}$ via

$$
\frac{\Sigma\left(s, g_{\mathrm{SF}}^{2}(L), a / L\right)-\sigma\left(s, g_{\mathrm{SF}}^{2}(L)\right)}{\sigma\left(s, g_{\mathrm{SF}}^{2}(L)\right)}=\sum_{n=1} g_{\mathrm{SF}}^{2 n}(L) \delta_{n}(s, a / L) .
$$

The $\delta$ 's hopefully give a hint about the magnitude of scaling violation in numerical simulations. Scaling for $\sigma$ in perturbation theory looks comparable for $N_{\mathrm{f}}=2$ and $N_{\mathrm{f}}=0$. This is shown in figure 2 for the two loop contribution $\delta_{2}(2, a / L)$.

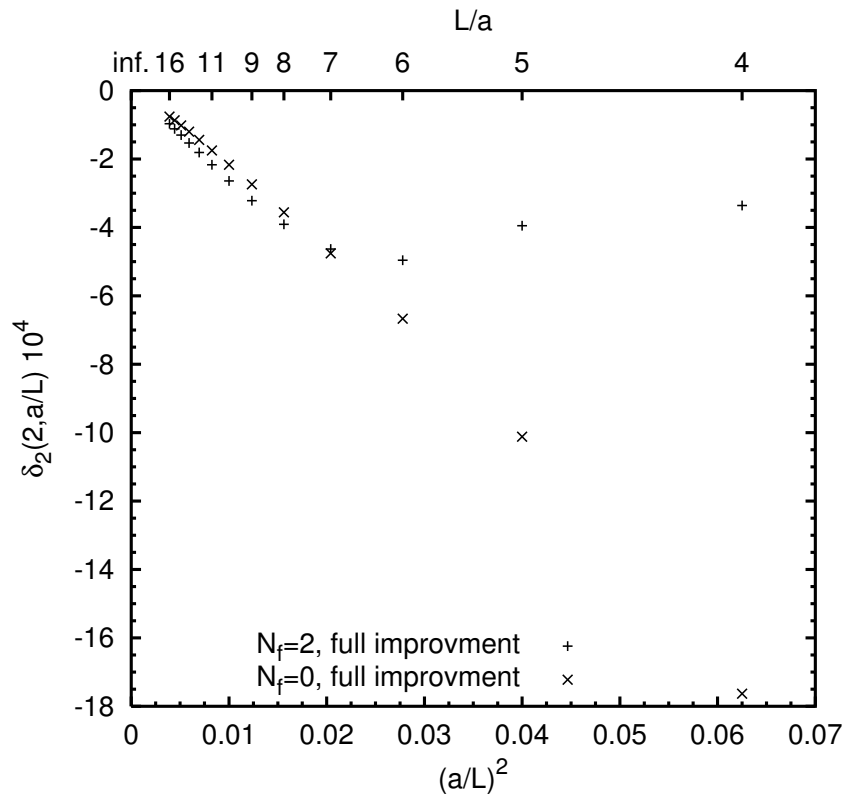

Figure 2: Two loop contribution to the scaling violations of the step scaling function $\sigma$. 


\section{Conclusion}

The two loop expansion of the Schrödinger functional with the SheikholeslamiWohlert fermion action is numerically demanding in comparison to the pure gauge case. For $\alpha_{\mathrm{SF}}$ the three loop $\beta$-function is now known and conversion to $\alpha \overline{\mathrm{MS}}$ is possible at two loops. For the dynamical simulations of the Schrödinger functional the perturbative results have small $\mathrm{O}(a)$ effects and do not indicate particular complications for the continuum extrapolation.

\section{References}

[1] S. Capitani, M. Lüscher, R. Sommer and H. Wittig, Nucl.Phys. B544 (1999) 669

[2] K. Jansen, C. Liu, M. Lüscher, H. Simma, S. Sint, R. Sommer, P.Weisz and U. Wolff, Phys. Lett. B372 (1996) 275

[3] A. Bode, P. Weisz and U. Wolff, Nucl. Phys. B540 (1999) 491

[4] M. Lüscher, S. Sint, R. Sommer and P. Weisz, Nucl.Phys. B478 (1996) 365

[5] S. Sint and R. Sommer, Nucl. Phys. B465 (1996) 71

[6] A. Bode, P. Weisz and U. Wolff, Nucl. Phys. B576 (2000) 517-539; Erratumibid. B600 (2001) 453; second Erratum to appear; hep-lat/9911018v3

[7] S. Capitani, M. Guagnelli, M. Lüscher, S. Sint, R. Sommer, P. Weisz and H. Wittig, Nucl. Phys. Proc. Suppl. 63 (1998) 796

[8] M. Lüscher and P. Weisz, Nucl. Phys. B452 (1995) 234

[9] C. Christou, A. Feo, H. Panagopoulos, Nucl. Phys. B525 (1998) 387; Erratum to appear; hep-lat/9801007 $\mathrm{v} 2$ 